PROCEEDINGS OF THE

AMERICAN MATHEMATICAL SOCIETY

Volume 125, Number 6, June 1997, Pages 1815-1822

S $0002-9939(97) 03741-6$

\title{
BROWNIAN FUNCTIONALS ON HYPERSURFACES IN EUCLIDEAN SPACE
}

\author{
KIMBERLY K. J. KINATEDER AND PATRICK MCDONALD
}

(Communicated by Richard T. Durrett)

\begin{abstract}
Using the first exit time for Brownian motion from a smoothly bounded domain in Euclidean space, we define two natural functionals on the space of embedded, compact, oriented, unparametrized hypersurfaces in Euclidean space. We develop explicit formulas for the first variation of each of the functionals and characterize the critical points.
\end{abstract}

\section{INTRODUCTION}

In this note we study several natural variational problems on the space of embedded, compact, oriented, unparametrized hypersurfaces in $\mathbb{R}^{d}$ which bound a connected open domain. We denote this space by $\mathcal{S} H\left(\mathbb{R}^{d}\right)$. Given $S \in \mathcal{S} H\left(\mathbb{R}^{d}\right)$, let $D=D(S)$ be the domain bounded by $S$ and let $|D|$ be the volume of $D$. Let $\tau_{S}$ be the first exit time of Brownian motion from $D(S)$; that is,

$$
\tau_{S}=\tau_{S}(\omega)=\inf \left\{t \geq 0: W_{t}(\omega) \in S\right\}
$$

where $W_{t}$ is $d$-dimensional Brownian motion.

We define a functional $\mathcal{A}: \mathcal{S} H\left(\mathbb{R}^{d}\right) \longrightarrow \mathbb{R}$ by

$$
\mathcal{A}(S)=\frac{1}{|D|} \int_{D} E_{x}\left(\tau_{S}\right) d x
$$

where $E_{x}\left(\tau_{S}\right)$ is the expected value of the random variable $\tau_{S}$, given that Brownian motion starts at $x$.

For $v_{0}$ an arbitrary constant, define $\mathcal{S} H_{v_{0}}\left(\mathbb{R}^{d}\right)$ by

$$
\mathcal{S} H_{v_{0}}\left(\mathbb{R}^{d}\right)=\left\{S \in \mathcal{S} H\left(\mathbb{R}^{d}\right):|D(S)|=v_{0}\right\} .
$$

We characterize the critical points of $\mathcal{A}$ in the following theorem.

Theorem 1.1. Let $\mathcal{A}: \mathcal{S} H_{v_{0}}\left(\mathbb{R}^{d}\right) \longrightarrow \mathbb{R}$ be defined as above. Then $\mathcal{A}$ is a smooth functional and the critical points of $\mathcal{A}$ are spheres bounding balls of volume $v_{0}$. Moreover, each critical point defines a global maximum.

We prove Theorem $\mathbf{1 . 1}$ by obtaining an explicit formula for the first variation of the functional $\mathcal{A}$ (see Corollary 3.1). Our strategy is to use Itô's formula to express the expected stopping time $E_{x}\left(\tau_{S}\right)$ in terms of a solution of a Poisson problem on $D(S)$. We then use Hadamard's classical approach to domain variation to obtain

Received by the editors August 9, 1995 and, in revised form, December 2, 1995.

1991 Mathematics Subject Classification. Primary 60J65, 58G32.

Key words and phrases. Brownian motion, exit times, variational calculus.

(C)1997 American Mathematical Society 
formulas for the variation of the functional $\mathcal{A}$. Finally, we use a rearrangement argument of Polya $[\mathrm{P}]$ to classify the critical points as global maxima.

Let $x_{0} \in \mathbb{R}^{d}$ and consider the subspaces

$$
\begin{aligned}
\mathcal{S} H\left(\mathbb{R}^{d}, x_{0}\right) & =\left\{S \in \mathcal{S} H\left(\mathbb{R}^{d}\right): x_{0} \in D(S)\right\}, \\
\mathcal{S} H_{v_{0}}\left(\mathbb{R}^{d}, x_{0}\right) & =\mathcal{S} H\left(\mathbb{R}^{d}, x_{0}\right) \cap \mathcal{S} H_{v_{0}}\left(\mathbb{R}^{d}\right) .
\end{aligned}
$$

Define a functional $\mathcal{E}: \mathcal{S} H\left(\mathbb{R}^{d}, x_{0}\right) \longrightarrow \mathbb{R}$ by

$$
\mathcal{E}(S)=E_{x_{0}}\left(\tau_{S}\right)
$$

The functional $\mathcal{A}$ restricts to $\mathcal{S} H\left(\mathbb{R}^{d}, x_{0}\right)$ and both $\mathcal{A}$ and $\mathcal{E}$ restrict to $\mathcal{S} H_{v_{0}}\left(\mathbb{R}^{d}, x_{0}\right)$. Indeed, the higher moments of the stopping time, $E_{x_{0}}\left(\tau_{S}^{n}\right)$, define smooth functionals on the space $\mathcal{S} H\left(\mathbb{R}^{d}, x_{0}\right)$. It is a theorem of Aizenman and Simon [AS] that the sphere centered at $x_{0}$ maximizes $E_{x_{0}}\left(\tau_{S}^{n}\right)$ for every $n$. A natural extension of this result would characterize the sphere as the only critical point of the various moment functionals (cf. Theorem 1.1). As a first step in this direction we develop a variational formula for the functional $\mathcal{E}: \mathcal{S} H_{v_{0}}\left(\mathbb{R}^{d}, x_{0}\right) \longrightarrow \mathbb{R}$ from which it is obvious that the sphere is a critical point. The converse statement depends on a symmetry result related to the fundamental result of Serrin [S] (cf. Corollary 3.2 and the proof of Theorem 1.1). This result is established in [FM].

We extend a special thanks to Steven J. Fromm for a careful reading of this manuscript. In addition, we thank the Referee for insightful comments which improved the exposition.

\section{BASIC RESULTS AND DEFINITIONS}

Let $(\Omega, \mathcal{B})$ be a measurable space and $\left\{P_{x}\right\}_{x \in \mathbb{R}^{d}}$ a family of probability measures on $(\Omega, \mathcal{B})$. Let $\left\{W_{t}\right\}_{t \geq 0}$ denote $d$-dimensional Brownian motion for which $P_{x}\left\{W_{0}=\right.$ $x\}=1$, for $x \in \mathbb{R}^{d}$.

Let $\mathcal{S} H\left(\mathbb{R}^{d}\right)$ be as in the introduction, and note that $\mathcal{S} H\left(\mathbb{R}^{d}\right)$ is a Fréchet manifold. Given a point $S \in \mathcal{S} H\left(\mathbb{R}^{d}\right)$ we can identify the tangent space at $S$ with the space of smooth vectorfields normal to $S$. The space of smooth vectorfields normal to $S$ can be naturally identified with the space of smooth functions on $S$ and we obtain an identification: $T_{S} \mathcal{S} H\left(\mathbb{R}^{d}\right) \simeq C^{\infty}(S)$. From its definition it is clear that $\mathcal{S} H\left(\mathbb{R}^{d}, x_{0}\right)$ is an open set in $\mathcal{S} H\left(\mathbb{R}^{d}\right)$. It is a Fréchet manifold with tangent space at $S$ given by $C^{\infty}(S)$.

Given $S \in \mathcal{S} H\left(\mathbb{R}^{d}\right)$ and $f \in C^{\infty}(S)$, a normal variation of $S$ in the direction of $f$ is a one-parameter family $\left\{S^{\epsilon}\right\} \subset \mathcal{S} H\left(\mathbb{R}^{d}\right)$,

$$
S^{\epsilon}=\left\{y \in \mathbb{R}^{d}: y=\sigma+\epsilon f(\sigma) \nu(\sigma), \sigma \in S\right\},
$$

where $\nu(\sigma)$ is the outward pointing unit normal vector to $S$ at $\sigma$. For $\epsilon$ sufficiently small, the map $F: S \longrightarrow S^{\epsilon}$ defined by $F(\sigma)=y$ is a diffeomorphism. Normal variations allow us to introduce coordinates in a neighborhood of a point $S \in$ $\mathcal{S} H\left(\mathbb{R}^{d}\right)$. The coordinates near $S$ are given by the space $C^{\infty}(S)$ and it is in these coordinates that our computations are carried out.

Given $S \in \mathcal{S} H\left(\mathbb{R}^{d}\right)$, the volume of $D(S)$ defines a smooth functional $|D|$ : $\mathcal{S} H\left(\mathbb{R}^{d}\right) \longrightarrow \mathbb{R}$. We say that a normal variation of $S$ in direction $f$ fixes volume if the variation of $|D|$ in the direction of $f$ (denoted $(\delta|D|) f$ ) vanishes. A simple computation establishes that a normal variation in direction $f$ fixes volume if and only 
if $\int_{S} f d \sigma=0$. We will henceforth denote the space of smooth mean-zero functions on $S$ by

$$
\mathcal{M}(S)=\left\{f \in C^{\infty}(S): \int_{S} f d \sigma=0\right\} .
$$

Note that the space $\mathcal{S} H_{v_{0}}\left(\mathbb{R}^{d}\right)$ is a Fréchet manifold with tangent space at $S \in$ $\mathcal{S} H_{v_{0}}\left(\mathbb{R}^{d}\right)$ identified with $\mathcal{M}(S)$. The space $\mathcal{S} H_{v_{0}}\left(\mathbb{R}^{d}, x_{0}\right)$ is an open set in $\mathcal{S} H_{v_{0}}\left(\mathbb{R}^{d}\right)$. It is also a Fréchet manifold with tangent space at $S$ identified with $\mathcal{M}(S)$.

As in the introduction, for $S \in \mathcal{S} H\left(\mathbb{R}^{d}\right)$, we define the first exit time of Brownian motion from $D(S)$ by $\tau_{S}=\tau_{S}(\omega)=\inf \left\{t: W_{t}(\omega) \in S\right\}$, where $\omega \in \Omega$. For each $x \in \mathbb{R}^{d}$, we will denote the expected value of a random variable $Y$ under the probability measure $P_{x}$ by $E_{x}(Y)$. In particular, for Brownian motion starting at the point $x$, the expected value of the stopping time $\tau_{S}$ is $E_{x}\left(\tau_{S}\right)=\int_{\Omega} \tau_{S} d P_{x}$.

Given an element $S \in \mathcal{S} H\left(\mathbb{R}^{d}\right)$ there is a useful relationship between the solution of a certain Poisson problem on the domain $D(S)$ and the expected value of the first exit time of Brownian motion from $D(S)$ starting at $x \in D(S)$. Indeed, suppose $u$ solves the problem

$$
\begin{aligned}
\Delta u & =-2 \text { on } D(S), \\
u & =0 \text { on } S .
\end{aligned}
$$

Using Itô's formula we have

$$
u\left(W_{\tau_{S}}\right)-u\left(W_{0}\right)=\int_{0}^{\tau_{S}} \nabla u\left(W_{s}\right) \cdot d W_{s}+\frac{1}{2} \int_{0}^{\tau_{S}} \Delta u\left(W_{s}\right) d s
$$

where the first random variable, $\int_{0}^{\tau_{S}} \nabla u\left(W_{s}\right) \cdot d W_{s}$, is a stochastic integral taken with respect to Brownian motion. From this it follows that

$$
\tau_{S}=u\left(W_{0}\right)+\int_{0}^{\tau_{S}} \nabla u\left(W_{s}\right) \cdot d W_{s},
$$

and taking expectations of both sides we obtain

$$
E_{x}\left(\tau_{S}\right)=u(x) .
$$

In particular, we can express the functional $\mathcal{A}: \mathcal{S} H\left(\mathbb{R}^{d}\right) \longrightarrow \mathbb{R}$ as

$$
\mathcal{A}(S)=\frac{1}{|D|} \int_{D} u(x) d x
$$

and the functional $\mathcal{E}: \mathcal{S} H\left(\mathbb{R}^{d}, x_{0}\right) \longrightarrow \mathbb{R}$ as

$$
\mathcal{E}(S)=u\left(x_{0}\right),
$$

where $u$ solves (2.1). This establishes that the functionals $\mathcal{A}$ and $\mathcal{E}$ are smooth.

\section{First VARiation}

Let $\alpha, \beta: \mathbb{R}^{d} \longrightarrow \mathbb{R}$ be smooth functions. Given a hypersurface $S \in \mathcal{S} H\left(\mathbb{R}^{d}\right)$, let $w=w_{\alpha, \beta}$ be the solution of the Poisson problem

$$
\begin{aligned}
\Delta w & =\alpha \text { on } D(S), \\
w & =\beta \text { on } S .
\end{aligned}
$$


Using $w$ we define smooth functionals $A_{\alpha, \beta}: \mathcal{S} H\left(\mathbb{R}^{d}\right) \longrightarrow \mathbb{R}$ and $F_{\alpha, \beta}: \mathcal{S} H\left(\mathbb{R}^{d}, x_{0}\right)$ $\longrightarrow \mathbb{R}$ by

$$
\begin{aligned}
A_{\alpha, \beta}(S) & =\frac{1}{|D|} \int_{D} w(x) d x, \\
F_{\alpha, \beta}(S) & =w\left(x_{0}\right) .
\end{aligned}
$$

Proposition 3.1. Let $S \in \mathcal{S} H\left(\mathbb{R}^{d}\right)$ and suppose $\delta A_{\alpha, \beta}: T_{S} \mathcal{S} H\left(\mathbb{R}^{d}\right) \longrightarrow \mathbb{R}$ is the variation of $A_{\alpha, \beta}$ at $S$. Then

$$
\begin{aligned}
\left(\delta A_{\alpha, \beta}\right) f= & \frac{1}{|D|}\left[-[(\delta|D|) f] A_{\alpha, \beta}(S)-\frac{1}{2} \int_{S} f(\sigma)\left(\frac{\partial \beta}{\partial \nu}(\sigma)-\frac{\partial w}{\partial \nu}(\sigma)\right) \frac{\partial u}{\partial \nu}(\sigma) d \sigma\right] \\
& +\frac{1}{|D|} \int_{S} f(\sigma) \beta(\sigma) d \sigma
\end{aligned}
$$

where $f \in C^{\infty}(S)$ is a tangent vector, d $\sigma$ is surface measure on $S, \nu$ is the outward pointing unit normal vector, $w(x)$ solves (3.1), and $u(x)$ solves (2.1).

Proof. Let $S \in \mathcal{S} H\left(\mathbb{R}^{d}, x_{0}\right)$ and suppose $f \in C^{\infty}(S)$. Let $S^{\epsilon}$ be a normal variation of $S$ in the direction of $f$ and denote $D\left(S^{\epsilon}\right)$ by $D^{\epsilon}$. Let $w$ be the solution of (3.1) and let $w_{\epsilon}$ be the solution of

$$
\begin{aligned}
\Delta w_{\epsilon} & =\alpha \text { on } D^{\epsilon}, \\
w_{\epsilon} & =\beta \text { on } S^{\epsilon} .
\end{aligned}
$$

To compute $\left(\delta \mathcal{A}_{\alpha, \beta}\right)(f)$, we compute

$$
\int_{D^{\epsilon}} w_{\epsilon}-\int_{D} w=\int_{D^{\epsilon} \cap D}\left(w_{\epsilon}-w\right)+\int_{D^{\epsilon} \backslash D} w_{\epsilon}-\int_{D \backslash D^{\epsilon}} w .
$$

The difference $v_{\epsilon}=w_{\epsilon}-w$ is harmonic on $D^{\epsilon} \cap D$, and we can use Green's Theorem to write

$$
\begin{aligned}
\int_{D^{\epsilon} \cap D}\left(w_{\epsilon}-w\right) \Delta u d x & =\int_{\partial\left(D^{\epsilon} \cap D\right)}\left[\left(w_{\epsilon}-w\right) \frac{\partial u}{\partial n}-u \frac{\partial\left(w_{\epsilon}-w\right)}{\partial n}\right] d z \\
& =\int_{\partial\left(D^{\epsilon} \cap D\right)}\left(w_{\epsilon}-w\right) \frac{\partial u}{\partial n} d z+o(\epsilon)
\end{aligned}
$$

where $u$ solves (2.1) and $n$ is the outward normal vector to $D^{\epsilon} \cap D$. The boundary $\partial\left(D^{\epsilon} \cap D\right)$ can be partitioned as $\partial\left(D^{\epsilon} \cap D\right)=S_{+} \cup S_{-}^{\epsilon}$, where

$$
\begin{aligned}
& S_{+}=\{\sigma \in S: f(\sigma) \geq 0\}, \\
& S_{-}=\{\sigma \in S: f(\sigma)<0\}, \\
& S_{-}^{\epsilon}=\left\{y=\sigma+\epsilon f(\sigma) \nu(\sigma) \in S^{\epsilon}: \sigma \in S_{-}\right\} .
\end{aligned}
$$

We write

$$
\int_{\partial\left(D^{\epsilon} \cap D\right)}\left(w_{\epsilon}-w\right) \frac{\partial u}{\partial n} d z=\int_{S_{+}}\left(w_{\epsilon}-w\right) \frac{\partial u}{\partial \nu} d \sigma+\int_{S_{-}^{\epsilon}}\left(w_{\epsilon}-w\right) \frac{\partial u}{\partial \nu_{\epsilon}} d z
$$

where $\nu_{\epsilon}$ is the outward normal to $S^{\epsilon}$ and $d z$ is surface measure. Expanding $w(\sigma+\epsilon f \nu)$ and $\beta(\sigma+\epsilon f \nu)$ near $\epsilon=0$, we obtain

$$
\begin{aligned}
& w(\sigma+\epsilon f \nu)-w(\sigma)=\epsilon f \frac{\partial w}{\partial \nu}+o(\epsilon), \\
& \beta(\sigma+\epsilon f \nu)-\beta(\sigma)=\epsilon f \frac{\partial \beta}{\partial \nu}+o(\epsilon) .
\end{aligned}
$$


Let $J_{\epsilon}$ be the Jacobian of the map $S \rightarrow S^{\epsilon}$ given by $\sigma \rightarrow y=\sigma+\epsilon f(\sigma) \nu(\sigma)$. Then, using (3.2) and (3.3), we have

$$
\begin{aligned}
\int_{S_{-}} & \left(w_{\epsilon}-w\right) \frac{\partial u}{\partial \nu_{\epsilon}} d z=\int_{S_{-}}(\beta(y)-w(y)) \frac{\partial u}{\partial \nu_{\epsilon}}(y) J_{\epsilon}(\sigma) d \sigma \\
= & \int_{S_{-}}\left[\beta(\sigma)-w(\sigma)+\epsilon f(\sigma)\left(\frac{\partial \beta}{\partial \nu}-\frac{\partial w}{\partial \nu}\right)\right] \frac{\partial u}{\partial \nu}(\sigma) J_{\epsilon}(\sigma) d \sigma+o(\epsilon) .
\end{aligned}
$$

Noting that $J_{\epsilon}(\sigma)=1+O(\epsilon)$, and that $\beta(\sigma)-w(\sigma)=0$ for $\sigma \in S$, we obtain

$$
\int_{S_{-}^{\epsilon}}\left(w_{\epsilon}-w\right) \frac{\partial u}{\partial \nu_{\epsilon}} d z=\epsilon \int_{S_{-}} f(\sigma)\left(\frac{\partial \beta}{\partial \nu}-\frac{\partial w}{\partial \nu}\right) \frac{\partial u}{\partial \nu} d \sigma+o(\epsilon) .
$$

Similarly,

$$
\int_{S_{+}}\left(w_{\epsilon}-w\right) \frac{\partial u}{\partial \nu} d \sigma=\epsilon \int_{S_{+}} f(\sigma)\left(\frac{\partial \beta}{\partial \nu}-\frac{\partial w}{\partial \nu}\right) \frac{\partial u}{\partial \nu} d \sigma+o(\epsilon)
$$

Hence,

$$
\int_{\partial\left(D^{\epsilon} \cap D\right)}\left(w_{\epsilon}-w\right) \frac{\partial u}{\partial n} d z=\epsilon \int_{S} f(\sigma)\left(\frac{\partial \beta}{\partial \nu}-\frac{\partial w}{\partial \nu}\right) \frac{\partial u}{\partial \nu} d \sigma+o(\epsilon) .
$$

Using (3.1), (3.2) and boundary normal coordinates,

$$
\begin{aligned}
\int_{D^{\epsilon} \backslash D} w_{\epsilon} & =\int_{S_{+}} \int_{0}^{\epsilon f(\sigma)} w_{\epsilon}(\sigma+r \nu) d r d \sigma+o(\epsilon) \\
& =\int_{S_{+}} \epsilon f(\sigma) \beta(\sigma) d \sigma+o(\epsilon)
\end{aligned}
$$

and

$$
\begin{aligned}
\int_{D \backslash D^{\epsilon}} w & =\int_{S_{-}} \int_{0}^{-\epsilon f(\sigma)} w(\sigma-r \nu) d r d \sigma+o(\epsilon) \\
& =\int_{S_{-}}-\epsilon f(\sigma) \beta(\sigma) d \sigma+o(\epsilon) .
\end{aligned}
$$

Thus,

$$
\int_{D^{\epsilon} \backslash D} w_{\epsilon}-\int_{D \backslash D^{\epsilon}} w=\epsilon \int_{S} f(\sigma) \beta(\sigma) d \sigma+o(\epsilon) .
$$

Combining (3.4) and (3.5) we have

$$
\begin{aligned}
\int_{D_{\epsilon}} w_{\epsilon} d x-\int_{D} w d x= & -\frac{\epsilon}{2} \int_{S} f(\sigma)\left(\frac{\partial \beta}{\partial \nu}(\sigma)-\frac{\partial w}{\partial \nu}(\sigma)\right) \frac{\partial u}{\partial \nu}(\sigma) d \sigma \\
& +\epsilon \int_{S} f(\sigma) \beta(\sigma) d \sigma+o(\epsilon) .
\end{aligned}
$$

Dividing by $\epsilon$ and passing to the limit, the proposition follows.

Setting $\alpha=-2$ and $\beta=0$, we obtain

Corollary 3.1. Let $S \in \mathcal{S} H\left(\mathbb{R}^{d}\right)$ and suppose $\delta \mathcal{A}: T_{S} \mathcal{S} H\left(\mathbb{R}^{d}\right) \longrightarrow \mathbb{R}$ is the variation of $\mathcal{A}$ at $S$. Then

$$
(\delta \mathcal{A}) f=\frac{1}{|D|}\left[-[(\delta|D|) f] \mathcal{A}(S)+\frac{1}{2} \int_{S} f\left(\frac{\partial u}{\partial \nu}\right)^{2} d \sigma\right]
$$


where $f \in C^{\infty}(S)$ is a tangent vector, d $\sigma$ is surface measure on $S, \nu$ is the outward pointing unit normal vector and $u(x)$ solves $(2.1)$.

Proof of Theorem 1.1. From Corollary 3.1 we see that the variation of the functional $\mathcal{A}: \mathcal{S H}_{v_{0}}\left(\mathbb{R}^{d}\right) \longrightarrow \mathbb{R}$ is given by

$$
(\delta \mathcal{A}) f=\frac{1}{2} \frac{1}{|D|} \int_{S} f\left(\frac{\partial u}{\partial \nu}\right)^{2} d \sigma
$$

where $f \in C^{\infty}(S)$ satisfies $\int_{S} f d \sigma=0$. Hence $\partial u / \partial \nu$ must be a constant at any critical point $S$. It follows that any critical point is a sphere, for if $u$ solves (2.1) and $\partial u / \partial \nu$ is constant, then $S$ must be a sphere by a result of Serrin [S].

To prove that spheres bounding balls of volume $v_{0}$ define global maxima for the functional $\mathcal{A}$, we use the notion of symmetric rearrangement. For the benefit of the reader, we recall the definition and basic facts concerning rearrangements.

Let $f: \mathbb{R}^{d} \rightarrow \mathbb{R}^{+}$and denote by $\chi_{\{f>\gamma\}}(x)$ the characteristic function of the set $\{x: f(x)>\gamma\}$. Define the radius function associated to $f, R: \mathbb{R}^{+} \rightarrow \mathbb{R}^{+}$, by

$$
\omega_{d} R(\gamma)^{d}=\int \chi_{\{f>\gamma\}} d \lambda_{d}
$$

where $\omega_{d}$ is the volume of the unit ball in $\mathbb{R}^{d}$ and $\lambda_{d}$ denotes $d$-dimensional Lebesgue measure. Define the symmetric rearrangement of $f$, denoted $f^{*}$, by

$$
f^{*}(x)=\int_{\gamma>0} \chi_{[0, R(\gamma)]}(|x|) d \gamma
$$

The following facts are well-known $[\mathrm{AL}]$ :

(1) $f^{*}$ is symmetric: $f^{*}(x)=f^{*}(z)$ if $|x|=|z|$;

(2) $f^{*}$ is decreasing in $|x|$;

(3) $f$ and $f^{*}$ are equimeasurable; that is, $\lambda_{d}\{x: f(x)>\gamma\}=\lambda_{d}\left\{x: f^{*}(x)>\gamma\right\}$ for every $\gamma>0$

(4) rearrangement preserves $L^{p}$ norms; that is,

$$
\|f\|_{p}=\left\|f^{*}\right\|_{p}
$$

(5) rearrangement is norm non-increasing on the Sobolev space $W^{1, p}$ (consisting of those functions $f$ which belong to $L^{p}\left(\mathbb{R}^{d}\right)$ and whose distribution gradients are functions belonging to $L^{p}\left(\mathbb{R}^{d}\right)$ ), that is,

$$
\|\nabla f\|_{p} \geq\left\|\nabla f^{*}\right\|_{p} .
$$

Given $S \in \mathcal{S} H_{v_{0}}\left(\mathbb{R}^{d}\right)$, let $D^{*}=D^{*}(S)$ be the ball centered at the origin of volume $v_{0}$. Note that $f^{*}$ is defined on $D^{*}$. Let $\mathcal{F}_{D}$ be the space defined by

$$
\mathcal{F}_{D}=\left\{f \in C^{\infty}(D(S)): f \geq 0, f(\sigma)=0, \forall \sigma \in S\right\} .
$$

There is a variational characterization of the integral $\int_{D} u d x$ : if $f$ is a minimizer for the quotient $\frac{\int_{D}|\nabla f|^{2} d x}{\left(\int_{D} f d x\right)^{2}}$ then $f=c u$, where $c$ is a constant and $u$ solves (2.1) [P]. Hence,

$$
2\left(\int_{D} u d x\right)^{-1}=\inf _{f \in \mathcal{F}_{D}} \frac{\int_{D}|\nabla f|^{2} d x}{\left(\int_{D} f d x\right)^{2}} .
$$


Using (3.6), (3.7), and (3.8), we have

$$
\begin{aligned}
2\left(\int_{D} u d x\right)^{-1}=\frac{\int_{D}|\nabla u|^{2} d x}{\left(\int_{D} u d x\right)^{2}} & \geq \frac{\int_{D^{*}}\left|\nabla u^{*}\right|^{2} d x}{\left(\int_{D^{*}} u^{*} d x\right)^{2}} \\
& \geq \inf _{f \in \mathcal{F}_{D^{*}}} \frac{\int_{D^{*}}|\nabla f|^{2} d x}{\left(\int_{D^{*}} f d x\right)^{2}}=2\left(\int_{D^{*}} v d x\right)^{-1}
\end{aligned}
$$

where $v$ solves $(2.1)$ for the ball $D^{*}$. This completes the proof of the theorem.

We now characterize the critical points of the functional $\mathcal{E}(S)=E_{x_{0}} \tau_{S}$.

Proposition 3.2. Let $S \in \mathcal{S} H\left(\mathbb{R}^{d}, x_{0}\right)$ and suppose $\delta F_{\alpha, \beta}: T_{S} \mathcal{S} H\left(\mathbb{R}^{d}, x_{0}\right) \longrightarrow \mathbb{R}$ denotes the variation of $F_{\alpha, \beta}$ at $S$. Then

$$
\left(\delta F_{\alpha, \beta}\right) f=\int_{S} f(\sigma)\left(\frac{\partial \beta}{\partial \nu}(\sigma)-\frac{\partial w}{\partial \nu}(\sigma)\right) \frac{\partial G}{\partial \nu}\left(x_{0}, \sigma\right) d \sigma
$$

where $f \in C^{\infty}(S)$ is a tangent vector, d $\sigma$ is surface measure on $S, \nu$ is the outward pointing unit normal vector, $G(x, y)$ is the Green's function for the domain $D(S)$, and $w(x)$ solves (3.1).

Proof. The proof proceeds as in the previous proposition. Let $S \in \mathcal{S} H\left(\mathbb{R}^{d}, x_{0}\right)$ and suppose $f, S^{\epsilon}, w$ and $w_{\epsilon}$ are as in the proof of Proposition 3.1. For convenience we consider only the case $f \leq 0$, for which $S^{\epsilon} \subset S$. (The details for general $f$ are similar to those in the proof of Proposition 3.1.) The difference $v_{\epsilon}=w_{\epsilon}-w$ is harmonic on $D\left(S^{\epsilon}\right)$ and we can use the Poisson representation formula to write

$$
v_{\epsilon}\left(x_{0}\right)=\int_{S^{\epsilon}}[\beta(y)-w(y)] \frac{\partial G_{\epsilon}}{\partial \nu_{\epsilon}}\left(x_{0}, y\right) d y
$$

where $G_{\epsilon}$ is the Green's function for the domain $D\left(S^{\epsilon}\right)$ and $\nu_{\epsilon}$ the corresponding normal vector. As in the proof of Proposition 3.1, we change variables and use the expansions (3.2) and (3.3). Noting that $J_{\epsilon}(\sigma)=1+O(\epsilon)$, that $G_{\epsilon}=G+O(\epsilon)$, and that $\beta(\sigma)-w(\sigma)=0$ for $\sigma \in S$, we substitute into (3.9) and obtain

$$
v_{\epsilon}\left(x_{0}\right)=\epsilon \int_{S} f(\sigma)\left[\frac{\partial \beta}{\partial \nu}(\sigma)-\frac{\partial w}{\partial \nu}(\sigma)\right] \frac{\partial G}{\partial \nu}\left(x_{0}, \sigma\right) d \sigma+o(\epsilon) .
$$

Dividing by $\epsilon$ and passing to the limit concludes the proof of the proposition.

Setting $\alpha=-2$ and $\beta=0$, we obtain

Corollary 3.2. Let $S \in \mathcal{S} H\left(\mathbb{R}^{d}, x_{0}\right)$ and suppose $\delta \mathcal{E}: T_{S} \mathcal{S} H\left(\mathbb{R}^{d}, x_{0}\right) \longrightarrow \mathbb{R}$ denotes the variation of $\mathcal{E}$ at $S$. Then

$$
(\delta \mathcal{E}) f=-\int_{S} f(\sigma) \frac{\partial u}{\partial \nu}(\sigma) \frac{\partial G}{\partial \nu}\left(x_{0}, \sigma\right) d \sigma
$$

where $f \in C^{\infty}(S)$ is a tangent vector, d $\sigma$ is surface measure on $S, \nu$ is the outward pointing unit normal vector, $G(x, y)$ is the Green's function for the domain $D(S)$, and $u(x)$ solves (2.1).

Remark. From Corollary 3.2 it is clear that $S$ is a critical point for the functional $\mathcal{E}$ on $\mathcal{S} H_{v_{0}}\left(\mathbb{R}^{d}, x_{0}\right)$ if and only if $\frac{\partial u}{\partial \nu} \frac{\partial G}{\partial \nu}$ is a constant. Moreover, the existence of a solution to the overdetermined problem $\Delta u=-2$ on $D, u=0$ and $\frac{\partial u}{\partial \nu} \frac{\partial G}{\partial \nu}=k$ on $S$ implies that the surface $S$ is a sphere [FM]. Thus, there is a unique critical point for the functional $\mathcal{E}$ at which the functional attains a maximum [AS]. 


\section{REFERENCES}

[AS] M. Aizenman and B. Simon, Brownian motion and Harnack inequality for Schrodinger operators, Comm. Pure and Appl. Math. 35 (1982), 209-273. MR 84a:35062

[AL] F. J. Almgren, Jr. and E. H. Lieb, Symmetric decreasing rearrangement is sometimes continuous, J. Amer. Math. Soc. 2 (1989), 683-773. MR 90f:49038

[FM] S. J. Fromm and P. McDonald, A symmetry problem from probability, Proc. Amer. Math. Soc. (to appear).

[GS] P. R. Garabedian and M. Schiffer, Convexity of domain functionals, J. Analyse Math. 2 (1953), 281-368. MR 15:627a

[P] G. Polya, Torsional rigidity, principal frequency, electrostatic capacity and symmetrization, Quart. Appl. Math. 6 (1948), 267-277. MR 10:206b

[S] J. Serrin, A symmetry problem in potential theory, Arch. Rat. Mech. and Anal. 43 (1971), 304-318. MR 48:11545

Department of Mathematics, Ohio State University, Columbus, Ohio 43210

Current address, K. K. J. Kinateder: Department of Mathematics, Wright State University, Dayton, Ohio 45435

E-mail address, K. K. J. Kinateder: kjk@euler.math.wright.edu

Current address, P. McDonald: Department of Mathematics, New College of University of South Florida, Sarasota, Florida 34243

E-mail address, P. McDonald: pmacdona@virtu.sar.usf.edu 